\title{
SPIRALS AND THE UNIVERSAL TEICHMÜLLER SPACE
}

\author{
BY \\ F. W. GEHRING $\left({ }^{1}\right)$ \\ University of Michigan, Ann Arbor, Michigan, U.S.A. \\ Dedicated to Professor L. V. Ahlfors on his seventieth birthday
}

\section{Introduction}

Suppose that $D$ is a simply connected domain of hyperbolic type in the extended complex plane $\overline{\mathbf{C}}=\mathbf{C} \cup\{\infty\}$. Then the hyperbolic or noneuclidean metric $\varrho_{D}$ in $D$ is given by

$$
\varrho_{D}(z)=\left(1-|g(z)|^{2}\right)^{-1}\left|g^{\prime}(z)\right|,
$$

where $g$ is any conformal mapping of $D$ onto the unit disk $\{z:|z|<1\}$. For each function $\varphi$ defined in $D$ we introduce the norm

$$
\|\varphi\|_{D}=\sup _{z \in D}|\varphi(z)| \varrho_{D}(z)^{-2}
$$

Next for each function $f$ which is locally univalent and meromorphic in $D$ we let $S_{f}$ denote the Schwarzian derivative of $f$. At finite points of $D$ which are not poles of $f$, $S_{f}$ is given by

$$
S_{f}=\left(\frac{f^{\prime \prime}}{f^{\prime}}\right)^{\prime}-\frac{1}{2}\left(\frac{f^{\prime \prime}}{f^{\prime}}\right)^{2}=\frac{f^{\prime \prime \prime}}{f^{\prime}}-\frac{3}{2}\left(\frac{f^{\prime \prime}}{f^{\prime}}\right)^{2},
$$

and the definition is extended to $\infty$ and the poles of $f$ by means of inversion.

Now let $L$ denote the lower half plane, $L=\{z=x+i y: y<0\}$, and let $B_{2}=B_{2}(L, 1)$ denote the complex Banach space of functions $\varphi$ analytic in $L$ with the norm

$$
\|\varphi\|=\|\varphi\|_{L}=\sup _{z \in L_{L}} 4 y^{2}|\varphi(z)|<\infty .
$$

Next let $S$ denote the family of functions $\varphi=S_{g}$ where $g$ is conformal in $L$, and let $T=T(\mathrm{I})$ denote the subfamily of those $\varphi=S_{g}$ where $g$ has a quasiconformal extension to $\overline{\mathbf{C}}$. Then

(1) This research was supported in part by a grant from the U.S. National Science Foundation, Grant MCS-77-02842. 
$\|\varphi\| \leqslant 6$ for all $\varphi \in S$ by [11], and hence $T \subset S \subset B_{2}$. The set $T$ is called the universal Teichmïller space. See [4], [5], [6], [7].

In a recent paper [8], the author established a result, which when combined with an extension theorem of Ahlfors [1], yields the following characterization of $T$.

THEOREM 1. T is the interior of $S$.

Theorem 1 is closely related to the following interesting open problem raised by Bers in [4], [5], [6], [7].

\section{QUESTION. Is $S$ the closure of $T$ ?}

The purpose of this paper is to answer this question in the negative by establishing the following result.

THEOREM 2. There exists a simply connected domain $D$ of hyperbolic type and a positive constant $\delta$ with the following property. If $f$ is conformal in $D$ and if $\left\|\mathcal{S}_{f}\right\|_{D} \leqslant \delta$, then $f(D)$ is not a Jordan domain.

COROLLARY. There exists a $\varphi$ in $S$ which does not lie in the closure of $T$.

Proof of Corollary. Let $D$ and $\delta$ be as in Theorem 2, and let $g$ be any conformal mapping of $L$ onto $D$. Then $\varphi=S_{g} \in S$. Choose $\psi \in S$ with $\|\psi-\varphi\| \leqslant \delta$. Then $\psi=S_{h}$, where $h$ is conformal in $L$. Set $f=h \circ g^{-1}$. Then from the composition law

$$
S_{h}(z)=S_{f}(g(z)) g^{\prime}(z)^{2}+S_{g}(z)
$$

it follows that

$$
\left\|S_{f}\right\|_{D}=\left\|S_{h}-S_{g}\right\|_{L}=\|\psi-\varphi\| \leqslant \delta .
$$

Hence $h(L)=f(D)$ is not a Jordan domain, $h$ does not have even a homeomorphic extension to $\bar{L}$ and $\psi \notin T$. We conclude that $\varphi$ is a point of $S$ which does not lie in the closure of $T$.

The domain $D$ in Theorem 2 can be described in a very explicit manner. Namely, $D=\overline{\mathbf{C}}-\gamma$, where $\gamma$ is the arc

$$
\gamma=\left\{z= \pm i e^{(-a+i) t}: t \in[0, \infty)\right\} \cup\{0\}
$$

and $a \in(0,1 / 8 \pi)$. Hence it is not difficult to derive an analytic expression for the conformal mapping $g$ of $L$ onto $D$, and $\varphi=S_{g}$ turns out to be a rational function.

The idea behind the proof of Theorem 2 is quite simple. For $a \in(0, \infty)$ let

$$
\alpha_{1}=\left\{z=e^{(-a+i) t}: t \in(0, \infty)\right\}, \quad \alpha_{2}=\left\{z:-z \in \alpha_{1}\right\}
$$


Then $\alpha_{1}$ and $\alpha_{2}$ are logarithmic spirals in $D$ which converge onto the point 0 from opposite sides of $\partial D$. Next suppose that $f$ is conformal in $D$ and fixes the points $1,-1, \infty$. As $\left\|S_{f}\right\|_{D}$ approaches $0, f$ converges to the identity mapping in $D$. Hence for $\left\|S_{f}\right\|_{D}$ small, $f$ maps $\alpha_{1}, \alpha_{2}$ onto a pair of disjoint open ares $\alpha_{1}^{*}, \alpha_{2}^{*}$ which spiral onto $f_{1}(0), f_{2}(0)$, the points which $f(z)$ approaches as $z \rightarrow 0$ from the two sides of $\partial D$. This assertion follows from Lemmas 3, 5, 6 and 8 .

Now the rate at which $\alpha_{1}$ and $\alpha_{2}$, and hence $\alpha_{1}^{*}$ and $\alpha_{2}^{*}$, spiral depends on $a$. If $a$ is sufficiently small, then $\alpha_{1}^{*}, \alpha_{2}^{*}$ will spiral very slowly onto $f_{1}(0), f_{2}(0)$. Since $\alpha_{1}^{*}, \alpha_{2}^{*}$ are disjoint, the points $f_{1}(0), f_{2}(0)$ will either coincide or be separated by a distance greater than a positive constant $d$. This is a consequence of Lemma 1 .

Finally if we make $\left\|S_{f}\right\|_{D}$ still smaller, we can arrange by Lemma 9 that $f_{1}(0), f_{2}(0)$ lie near 0 and hence within distance $d$ of each other. Then $f_{1}(0)$ and $f_{2}(0)$ will coincide and $f(D)$ will not be a Jordan domain.

The complete proof for Theorem 2 is given in section 3 . As indicated above, it depends on a number of results for a class of spirals. These are established in section 2.

\section{Spirals}

We derive here the results on spirals which will be needed in the proof of Theorem 2 .

Definition. Suppose that $\alpha$ is an open arc in $\mathbf{C}$, that $z_{1}, z_{2} \in \mathbf{C}$ and that $b \in(1, \infty)$. We say that $\alpha$ is a spiral from $z_{1}$ onto $z_{2}$ if $\alpha$ has the representation

$$
z=z(t)=\left(z_{1}-z_{2}\right) r(t) e^{i t}+z_{2}, \quad t \in(0, \infty)
$$

where $r(t)$ is positive and continuous with

$$
\lim _{t \rightarrow 0} r(t)=1, \quad \lim _{t \rightarrow \infty} r(t)=0 .
$$

We say that $\alpha$ is a $b$-spiral if, in addition,

$$
\left|z\left(t_{1}\right)-z_{2}\right| \leqslant b\left|z\left(t_{2}\right)-z_{2}\right|
$$

for all $t_{1}, t_{2} \in(0, \infty)$ with $\left|t_{1}-t_{2}\right| \leqslant 2 \pi$.

Example. Suppose that $a>0$ and that $\alpha$ is the analytic open arc

$$
z=e^{(-a+i) t}, \quad t \in(0, \infty) \text {. }
$$

Then $\alpha$ is an $e^{2 \pi a}$-spiral from 1 onto 0 and

$$
k(z)|z|=\left(a^{2}+1\right)^{-\frac{1}{2}}, \quad \frac{d k}{d s}(z)|z|^{2}=a\left(a^{2}+1\right)^{-1}
$$


for all $z \in \alpha$, where $k$ denotes the curvature and $s$ the arclength of $\alpha$ taken in the direction from 1 to 0 .

Proposition 1. If $\alpha$ is a spiral from $z_{1}$ onto $z_{2}$ with the representation (2), then

$$
\left|z(t+2 \pi)-z_{2}\right|<\left|z(t)-z_{2}\right|
$$

for $t \in(0, \infty)$.

Proof. Let $A$ denote the set of $t \in(0, \infty)$ for which (6) holds and let $B=(0, \infty)-A$. Since $\alpha$ is an open arc, $B$ is the set of $t \in(0, \infty)$ for which the inequality in (6) is reversed. Hence $A$ and $B$ are both open. If $B \neq \varnothing$, then $B=(0, \infty)$ and

$$
\left|z(2 n \pi)-z_{2}\right| \geqslant\left|z(2 \pi)-z_{2}\right|>0
$$

for all integers $n \geqslant 1$ contradicting (3). Thus $A=(0, \infty)$.

PROposition 2. If $\alpha$ is a b-spiral from $z_{1}$ onto $z_{2}$ and if $f$ is a conformal similarity mapping, then $f(\alpha)$ is a b-spiral from $f\left(z_{1}\right)$ onto $f\left(z_{2}\right)$.

Proof. This is an immediate consequence of the above definition.

The proof of Theorem 2 is based on a simple geometric fact. Namely that when $b \in(1,2)$, the two points, onto which a pair of disjoint $b$-spirals converge, must either coincide or be separated by a distance greater than $\frac{1}{2} b^{-2}$ times the diameter of the smaller spiral. This observation is an immediate consequence of the following result.

LEMMA 1. Suppose that $\alpha$ is a b-spiral from $z_{1}$ onto $z_{2}$, that $\beta$ is a b-spiral from $w_{1}$ onto $w_{2}$ and that $\alpha \cap \beta=\varnothing$. If $b \in(1,2)$, then either $z_{2}=w_{2}$ or

$$
\left|z_{2}-w_{2}\right|>\frac{1}{b} \min \left(\left|z_{1}-z_{2}\right|,\left|w_{1}-w_{2}\right|\right)
$$

Proof. Suppose otherwise. Then

$$
0<\left|z_{2}-w_{2}\right| \leqslant \frac{1}{b} \min \left(\left|z_{1}-z_{2}\right|,\left|w_{1}-w_{2}\right|\right)
$$

If $\alpha$ has the representation (2), then $\arg \left(z\left(t_{0}\right)-z_{2}\right)=\arg \left(w_{2}-z_{2}\right)$ for some $t_{0} \in(0,2 \pi]$, and we obtain

$$
\left|z\left(t_{0}\right)-z_{2}\right| \geqslant \frac{1}{b} \lim _{t \rightarrow 0}\left|z(t)-z_{2}\right|=\frac{1}{b}\left|z_{1}-z_{2}\right| \geqslant\left|w_{2}-z_{2}\right|
$$


from (3), (4) and (7). For each integer $m \geqslant 0$ let $t_{1}=t_{0}+2 m \pi$. Then $\left|z\left(t_{1}\right)-z_{2}\right|$ decreases to 0 as $m \rightarrow \infty$, and we can fix $m$ so that

$$
\left\{\begin{array}{l}
\arg \left(z\left(t_{1}\right)-z_{2}\right)=\arg \left(w_{2}-z_{2}\right)=\arg \left(z\left(t_{1}+2 \pi\right)-z_{2}\right) \\
\left|z\left(t_{1}\right)-z_{2}\right| \geqslant\left|w_{2}-z_{2}\right|>\left|z\left(t_{1}+2 \pi\right)-z_{2}\right|
\end{array}\right.
$$

Similarly if $\beta$ has the representation $w=w(u), u \in(0, \infty)$, then we can choose $u_{1} \in(0, \infty)$ so that

$$
\left\{\begin{array}{l}
\arg \left(w\left(u_{1}\right)-w_{2}\right)=\arg \left(z_{2}-w_{2}\right)=\arg \left(w\left(u_{1}+2 \pi\right)-w_{2}\right) \\
\left|w\left(u_{1}\right)-w_{2}\right| \geqslant\left|z_{2}-w_{2}\right|>\left|w\left(u_{1}+2 \pi\right)-w_{2}\right|
\end{array}\right.
$$

Now let $\lambda$ denote the line through $z_{2}$ and $w_{2}$ directed from $z_{2}$ to $w_{2}$. Then (8) and (9) imply that $z\left(t_{1}\right), z\left(t_{1}+2 \pi\right), w\left(u_{1}\right), w\left(u_{1}+2 \pi\right)$ lie on $\lambda$, that $w\left(u_{1}\right)$ precedes or coincides with $z_{2}$, that $z\left(t_{1}\right)$ coincides with or follows $w_{2}$, and that $z\left(t_{1}+2 \pi\right)$ and $w\left(u_{1}+2 \pi\right)$ lie between $z_{2}$ and $w_{2}$. We claim that

To see this set

$$
\left|z\left(t_{1}+2 \pi\right)-z_{2}\right| \leqslant\left|w\left(u_{1}+2 \pi\right)-z_{2}\right|
$$

$$
\begin{aligned}
& A=\left\{z=s\left(z(t)-z_{2}\right)+z_{2}: s \in(0,1), t \in\left(t_{1}+\pi, t_{1}+3 \pi\right)\right\}, \\
& B=\left\{z=s\left(z(t)-z_{2}\right)+z_{2}: s \in(1, \infty), t \in\left(t_{1}+\pi, t_{1}+3 \pi\right)\right\}, \\
& \alpha_{1}=\left\{z=z(t): t \in\left(t_{1}+\pi, t_{1}+3 \pi\right)\right\} \subset \alpha, \\
& \beta_{1}=\left\{z=w(u): u \in\left(u_{1}+2 \pi, \infty\right)\right\} \subset \beta, \\
& \lambda_{1}=\left\{z=s\left(z\left(t_{1}+\pi\right)-z_{2}\right)+z_{2}: s \in[0, \infty)\right\} \subset \lambda .
\end{aligned}
$$

Then $A$ and $B$ are open and disjoint, $\beta_{1}$ joins $w\left(u_{1}+2 \pi\right)$ to $w_{2} \in B$ in $\mathbf{C}$, and

$$
\mathbf{C}=A \cup B \cup \alpha_{1} \cup \lambda_{1}
$$

From Proposition 1 it follows that

$$
\beta_{1} \cap\left(\alpha_{1} \cup \lambda_{1}\right)=\beta_{1} \cap \lambda_{1}=\varnothing
$$

and hence that $\beta_{1} \subset B$. Thus $w\left(u_{1}+2 \pi\right) \notin A$ and we obtain (10).

Finally since $\alpha$ and $\beta$ are $b$-spirals, Proposition 1 and (10) yield

$$
\begin{aligned}
\left|z\left(t_{1}\right)-z_{2}\right| & \leqslant b\left|z\left(t_{1}+2 \pi\right)-z_{2}\right| \leqslant b\left|w\left(u_{1}+2 \pi\right)-z_{2}\right| \\
& \leqslant b\left|w\left(u_{1}\right)-w\left(u_{1}+2 \pi\right)\right| \\
& =b\left(\left|w\left(u_{1}\right)-w_{2}\right|-\left|w\left(u_{1}+2 \pi\right)-w_{2}\right|\right) \\
& \leqslant(b-1)\left|w\left(u_{1}\right)-w_{2}\right|<\left|w\left(u_{1}\right)-w_{2}\right| .
\end{aligned}
$$


Next we can reverse the roles of $\alpha$ and $\beta$ in the above argument to obtain

$$
\left|w\left(u_{1}\right)-w_{2}\right|<\left|z\left(t_{1}\right)-z_{2}\right|
$$

This contradiction shows that (7) cannot hold, completing the proof of Lemma 1.

We derive next in Lemmas 2 and 3 conditions, similar to (5), which guarantee that an analytic open arc is a spiral or a $b$-spiral, respectively. By Proposition 2, we may restrict our attention to the case where the arc has 1 and 0 as its endpoints.

LEMMA 2. Suppose that $c, d \in(0, \infty)$, that $\alpha$ is an analytic open arc with 1 and 0 as endpoints, and that

$$
k(z)|z| \geqslant c, \quad \frac{d k}{d s}(z)|z|^{2} \geqslant d
$$

for $z \in \alpha$, where $s$ is taken in the direction from 1 to 0 . Then $\alpha$ is a rectifiable spiral from 1 onto 0.

Proof. For each $z \in \alpha$ let $\varrho(z)$ and $C(z)$ denote the radius and circle of curvature for $\alpha$ at $z$. Since $k$ is positive and increasing in $s$, the part of $\alpha$ from $z$ to 0 must lie inside $C(z)$ by a theorem due to A. Kneser. (See p. 48 in [9].) Hence

$$
|z| \leqslant 2 \varrho(z)=\frac{2}{k(z)}, \quad-\frac{d \varrho}{d s}(z)=\frac{\frac{d k}{d s}(z)}{k(z)^{2}} \geqslant \frac{d}{4}
$$

for $z \in \alpha$. If $\beta$ is any closed subarc of $\alpha$ from $w_{1}$ to $w_{2}$, then

$$
l(\beta)=\int_{\beta} d s \leqslant \frac{4}{d} \int_{\beta}\left(-\frac{d \varrho}{d s}\right) d s<\frac{4}{d} \varrho\left(w_{1}\right) \leqslant \frac{4}{c d}\left|w_{1}\right|,
$$

and hence $\alpha$ is rectifiable with length

$$
l=l(\alpha)=\sup _{\beta \subset \alpha} l(\beta) \leqslant \frac{4}{c d} .
$$

Let $s$ denote the arclength of $\alpha$ from 1 to $z$, let $z=z(s), s \in(0, l)$, denote the corresponding parametrization for $\alpha$, and choose a continuous branch of $\log z(s)$ so that $\log z(s) \rightarrow 0$ as $s \rightarrow 0$. Then $t(s)=\operatorname{Im}(\log z(s))$ is continuously differentiable with

$$
t^{\prime}(s)=\operatorname{Im}\left(\frac{z^{\prime}(s)}{z(s)}\right)
$$

Suppose that $t^{\prime}\left(s_{0}\right)=0$ for some $s_{0} \in(0, l)$. Then $z^{\prime}\left(s_{0}\right)=a z\left(s_{0}\right)$ where $a$ is a real constant. This implies that the circle of curvature $C\left(z\left(s_{0}\right)\right)$ is tangent to the ray from 0 through 
$z\left(s_{0}\right)$ and hence that $C\left(z\left(s_{0}\right)\right)$ cannot contain the point 0 , thus contradicting the above mentioned theorem of Kneser. We conclude that

$$
t^{\prime}(s)=\operatorname{Im}\left(\frac{z^{\prime}(s)}{z(s)}\right) \neq 0
$$

for $s \in(0, l)$ and hence that $t(s)$ is a strictly monotone function of $s$ in $(0, l)$.

By (11) we can choose a continuous branch of $\log \left(z^{\prime}(s) / z(s)\right)$ such that

$$
|\theta(s)|<\pi, \quad \theta(s)=\operatorname{Im}\left(\log \frac{z^{\prime}(s)}{z(s)}\right)
$$

in $(0, l)$. Then

$$
\varphi(s)=t(s)+\theta(s)=\operatorname{Im}\left(\log z^{\prime}(s)\right)
$$

determines the angle of inclination for the tangent vector $z^{\prime}(s)$ and

$$
\varphi^{\prime}(s)=k(z(s)) \geqslant c|z(s)|^{-1} \geqslant c(l-s)^{-1}
$$

for $s \in(0, l)$. If $s_{0} \in(0, l)$, then

$$
\varphi(s)-\varphi\left(s_{0}\right) \geqslant \int_{s_{0}}^{s} c(l-s)^{-1} d s=c \log \frac{l-s_{0}}{l-s}
$$

for $s \in\left(s_{0}, l\right)$, and $\varphi(s) \rightarrow \infty$ as $s \rightarrow l$. Thus $t(s) \rightarrow \infty$ as $s \rightarrow l$ by (12) and (13). Since $t(s) \rightarrow 0$ as $s \rightarrow 0$, we conclude that $s$ is a strictly increasing function of $t, s=s(t)$, in $(0, \infty)$. Set $r(t)=$ $|z(s(t))|$. Then

$$
z=r(t) e^{i t}, \quad t \in(0, \infty)
$$

is a representation for $\alpha$ which shows that $\alpha$ is a spiral from 1 onto 0 .

LEMMA 3. Suppose that $c_{1}, c_{2}, d_{1}, d_{2} \in(0, \infty)$ and that $4 \pi d_{2}<c_{1}^{2}$. Suppose also that $\alpha$ is an analytic open arc with 1 and 0 as endpoints and that

$$
c_{1} \leqslant k(z)|z| \leqslant c_{2}, \quad d_{1} \leqslant \frac{d k}{d s}(z)|z|^{2} \leqslant d_{2}
$$

for $z \in \alpha$, where $s$ is taken in the direction from 1 to 0 . Then $\alpha$ is a rectifiable b-spiral from 1 onto 0 , where

$$
b=\frac{c_{1} c_{2}}{c_{1}^{2}-4 \pi d_{2}}>1
$$

Proof. Lemma 2 implies that $\alpha$ is a rectifiable spiral from 1 onto 0 with the representation

$$
z=z(t)=r(t) e^{i t}, \quad t \in(0, \infty)
$$


It remains only to prove that $\left|z\left(t_{1}\right)\right| \leqslant b\left|z\left(t_{2}\right)\right|$ for all $t_{1}, t_{2} \in(0, \infty)$ with $\left|t_{1}-t_{2}\right| \leqslant 2 \pi$. Let $\varrho(z)$ denote the radius of curvature for $\alpha$ at $z$. Then since

$$
|z| \leqslant c_{2} \varrho(z) \leqslant \frac{c_{2}}{c_{1}}|z|
$$

it suffices to show that

$$
\varrho\left(z\left(t_{1}\right)\right) \leqslant \frac{c_{1}}{c_{2}} b \varrho\left(z\left(t_{2}\right)\right)
$$

for all such $t_{1}, t_{2}$.

Fix $t_{1}, t_{2} \in(0, \infty)$ with $\left|t_{1}-t_{2}\right| \leqslant 2 \pi$ and for $j=1,2$ let $z_{j}=z\left(t_{j}\right), s_{j}=s\left(t_{j}\right), \theta_{j}=\theta\left(s_{j}\right)$ and $\varphi_{j}=\varphi\left(s_{j}\right)$ where $\theta(s)$ and $\varphi(s)$ are as in the proof of Lemma 3 . Since

$$
0<-\frac{d \varrho}{d s}(z)=\frac{\frac{d k}{d s}(z)}{k(z)^{2}} \leqslant \frac{d_{2}}{c_{1}^{2}}
$$

for $z \in \alpha, \varrho(z)$ is decreasing as a function of $s$. Suppose that $s_{2} \leqslant s_{1}$. Then

$$
\varrho\left(z_{1}\right) \leqslant \varrho\left(z_{2}\right)<\frac{c_{1}}{c_{2}} b \varrho\left(z_{2}\right)
$$

and (14) holds. Suppose next that $s_{1}<s_{2}$. Then

$$
\varrho\left(z_{1}\right)-\varrho\left(z_{2}\right)=\int_{s_{1}}^{s_{2}}\left(-\frac{d \varrho}{d s}\right) d s \leqslant \frac{d_{2}}{c_{1}^{2}}\left(s_{2}-s_{1}\right)
$$

while

$$
s_{2}-s_{1}=\int_{\varphi_{1}}^{\varphi_{2}}\left(\frac{d s}{d \varphi}\right) d \varphi=\int_{\varphi_{1}}^{\varphi_{2}} \varrho d \varphi \leqslant \varrho\left(z_{1}\right)\left|\varphi_{2}-\varphi_{1}\right|
$$

Then (12) and (13) imply that

and we obtain

$$
\left|\varphi_{2}-\varphi_{1}\right| \leqslant\left|t_{2}-t_{1}\right|+\left|\theta_{2}-\theta_{1}\right| \leqslant 4 \pi
$$

$$
\varrho\left(z_{1}\right)-\varrho\left(z_{2}\right) \leqslant \frac{4 \pi d_{2}}{c_{1}^{2}} \varrho\left(z_{1}\right)
$$

from which (14) again follows. Hence the proof is complete.

We conclude this section with a result similar to Proposition 2. It implies that the image of a logarithmic spiral under a conformal mapping, which is nearly a similarity, is again a spiral. We require first the following result. 
LEMMA 4. Suppose that $\alpha$ is an analytic arc with the representation $z=z(t)$ where $z^{\prime}(t) \neq 0$, and suppose that $f$ maps a neighborhood of $\alpha$ conformally into C. Then $\alpha^{*}=f(\alpha)$ is an analytic arc with the representation $w=f \circ z(t)$ and

$$
\begin{gathered}
k^{*}(f(z))\left|f^{\prime}(z)\right|-k(z)=\operatorname{Im}\left(\frac{f^{\prime \prime}(z)}{f^{\prime}(z)} \frac{z^{\prime}(t)}{\left|z^{\prime}(t)\right|}\right), \\
\frac{d k^{*}}{d s^{*}}(f(z))\left|f^{\prime}(z)\right|^{2}-\frac{d k}{d s}(z)=\operatorname{Im}\left(S_{f}(z) \frac{z^{\prime}(t)^{2}}{\left|z^{\prime}(t)\right|^{2}}\right),
\end{gathered}
$$

where $k, k^{*}$ denote the curvatures and $s, s^{*}$ the arclengths of $\alpha, \alpha^{*}$ in the direction of increasing $t$.

Proof. If $w(t)=f \circ z(t)$, then $w^{\prime}(t)=f^{\prime}(z) z^{\prime}(t) \neq 0$ and

$$
\frac{w^{\prime \prime}(t)}{w^{\prime}(t)}-\frac{z^{\prime \prime}(t)}{z^{\prime}(t)}=\frac{f^{\prime \prime}(z)}{f^{\prime}(z)} z^{\prime}(t), \quad S_{w}(t)-S_{z}(t)=S_{f}(z) z^{\prime}(t)^{2},
$$

where $z=z(t)$ and where $S_{w}$ and $S_{z}$ are defined exactly as in (1) with the differentiation now taken with respect to the real variable $t$. Then

$$
k^{*}(w)\left|f^{\prime}(z)\right|-k(z)=\operatorname{Im}\left(\frac{w^{\prime \prime}(t)}{w^{\prime}(t)}-\frac{z^{\prime \prime}(t)}{z^{\prime}(t)}\right)\left|z^{\prime}(t)\right|^{-1}
$$

by elementary differential geometry and

$$
\frac{d k^{*}}{d s^{*}}(w)\left|f^{\prime}(z)\right|^{2}-\frac{d k}{d s}(z)=\operatorname{Im}\left(S_{w}(t)-S_{z}(t)\right)\left|z^{\prime}(t)\right|^{-2}
$$

by Exercise 3 on p. 21 of [3]. The desired conclusion now follows from (15), (16) and (17).

LemMa 5. Suppose that $b, c_{1}, c_{2}, d_{1}, d_{2}$ and $\alpha$ are as in Lemma 3 and that $b^{*} \in(b, \infty)$. Then there exists an $\varepsilon>0$, depending only on $b^{*}, c_{1}, c_{2}, d_{1}, d_{2}$, with the following property. If $f$ maps a neighborhood of $\alpha$ conformally into $\mathrm{C}$, if $f(z) \rightarrow 1$ and 0 as $z \rightarrow 1$ and 0 on $\alpha$, and if

$$
\left|\frac{z f^{\prime}(z)}{f(z)}-1\right| \leqslant \varepsilon, \quad\left|\frac{z^{2} f^{\prime \prime}(z)}{f(z)}\right| \leqslant \varepsilon, \quad\left|\frac{z^{3} f^{\prime \prime \prime}(z)}{f(z)}\right| \leqslant \varepsilon
$$

for $z \in \alpha$, then $\alpha^{*}=f(\alpha)$ is a $b^{*}$-spiral from $\mathrm{I}$ onto 0 .

Proof. By hypothesis we can choose $\eta \in\left(0, \min \left(c_{1}, d_{1}\right)\right)$ so that

$$
4 \pi\left(d_{2}+\eta\right)<\left(c_{1}-\eta\right)^{2}, \quad \frac{\left(c_{1}-\eta\right)\left(c_{2}+\eta\right)}{\left(c_{1}-\eta\right)^{2}-4 \pi\left(d_{2}+\eta\right)} \leqslant b^{*}
$$


Fix $\varepsilon \in\left(0, \frac{1}{2}\right)$ so that $\left(4+2 c_{2}\right) \varepsilon \leqslant \eta$ and $\left(20+6 d_{2}\right) \varepsilon \leqslant \eta$, and suppose that $f$ satisfies the hypotheses of Lemma 5. Then $\alpha^{*}=f(\alpha)$ is an analytic open arc with 1 and 0 as endpoints. If $w \in \alpha^{*}$, then $z=f^{-1}(w) \in \alpha$ and (18) implies that

$$
|| \frac{f(z)}{f^{\prime}(z)}|-| z|| \leqslant 2 \varepsilon|z|, \quad\left|\frac{z f^{\prime \prime}(z)}{f^{\prime}(z)}\right| \leqslant 2 \varepsilon, \quad\left|z^{2} S_{f}(z)\right| \leqslant 5 \varepsilon .
$$

Hence we obtain the inequalities

$$
\begin{aligned}
\left|k^{*}(w)\right| w|-k(z)| z|| & \leqslant\left|\frac{f(z)}{f^{\prime}(z)}\right|\left|k^{*}(f(z))\right| f^{\prime}(z)|-k(z)|+|| \frac{f(z)}{f^{\prime}(z)}|-| z|| k(z) \\
& \leqslant 2|z|\left|\frac{f^{\prime \prime}(z)}{f^{\prime}(z)}\right|+2 \varepsilon|z| k(z) \leqslant \eta
\end{aligned}
$$

and

$$
\begin{aligned}
\left.\left|\frac{d k^{*}}{d s^{*}}(w)\right| w\right|^{2}-\frac{d k}{d s}(z)|z|^{2} \mid & \leqslant\left.\left|\frac{f(z)}{f^{\prime}(z)}\right|^{2}\left|\frac{d k^{*}}{d s^{*}}(f(z))\right| f^{\prime}(z)\right|^{2}-\frac{d k}{d s}(z)|+|\left|\frac{f(z)}{f^{\prime}(z)}\right|^{2}-|z|^{2} \mid \frac{d k}{d s}(z) \\
& \leqslant 4|z|^{2}\left|S_{f}(z)\right|+6 \varepsilon|z|^{2} \frac{d k}{d s}(z) \leqslant \eta
\end{aligned}
$$

from Lemma 4, where $k^{*}$ and $s^{*}$ denote the curvature and arclength of $\alpha^{*}$. Thus

$$
c_{1}-\eta \leqslant k^{*}(w)|w| \leqslant c_{2}+\eta, \quad d_{1}-\eta \leqslant \frac{d k^{*}}{d s^{*}}(w)|w|^{2} \leqslant d_{2}+\eta
$$

for $w \in \alpha^{*}$, and the desired conclusion follows from Lemma 3.

\section{Proof of Theorem 2}

For each $a \in(0, \infty)$ let

$$
\begin{aligned}
& \alpha_{1}=\left\{z=e^{(-a+i) t}: t \in(0, \infty)\right\}, \quad \alpha_{2}=\left\{z:-z \in \alpha_{1}\right\} \\
& \beta=\left\{z= \pm i e^{(-a+i) t}: t \in(-\infty, \infty)\right\} \cup\{0, \infty\} \\
& \gamma=\{z: z \in \beta,|z| \leqslant 1\}
\end{aligned}
$$

Then $\beta$ is a Jordan curve which separates $\alpha_{1}$ and $\alpha_{2}$. Let $D_{j}$ denote the component of $\overline{\mathbf{C}}-\beta$ which contains $\alpha_{j}$ and set $D=\overline{\mathbf{C}}-\gamma$. Then $D$ is a simply connected domain of hyperbolic type which contains $D_{1} \cup D_{2}$ and hence $\alpha_{1} \cup \alpha_{2}$.

Now suppose that $a \in(0,1 / 8 \pi)$ and that $f$ is conformal in $D$. We shall show that there exists a $\delta=\delta(a)>0$ such that $f(D)$ is not a Jordan domain whenever $\left\|S_{f}\right\|_{D} \leqslant \delta$; for this we may clearly assume that $f$ is normalized so that it fixes the points $1,-1, \infty$. The argument 
then consists of three steps. First in Lemma 8 we show there exists a $\delta_{2}>0$ such that $f\left(\alpha_{1}\right)$ and $f\left(\alpha_{2}\right)$ are $b^{*}$-spirals with $b^{*} \in(1,2)$ whenever $\left\|S_{f}\right\|_{D} \leqslant \delta_{2}$. Next in Lemma 9 we show there exists a $\delta_{3}>0$ such that the points onto which $f\left(\alpha_{1}\right)$ and $f\left(\alpha_{2}\right)$ converge must lie in $\left\{z:|z| \leqslant \frac{1}{5}\right\}$ whenever $\left\|S_{f}\right\|_{D} \leqslant \delta_{3}$. Finally set $\delta=\min \left(\delta_{2}, \delta_{3}\right)$. Then Lemma 1 implies that $f\left(\alpha_{1}\right)$ and $f\left(\alpha_{2}\right)$ converge onto the same point and hence that $f(D)$ is not a Jordan domain whenever $\left\|S_{f}\right\|_{D} \leqslant \delta$.

We begin with an application of Ahlfors' extension theorem [1] to the domains $D_{1}$ and $D_{2}$.

LEMMA 6. There exists a $\delta_{1}=\delta_{1}(a)>0$ with the following property. If $f$ is conformal in $D$ and if $\left\|S_{f}\right\|_{D} \leqslant \delta_{1}$, then for $j=1,2$ the mapping $f_{j}=f \mid D_{j}$ has a quasiconformal extension $g_{j}$ to $\overline{\mathbf{C}}$ and

$$
K\left(g_{j}\right) \leqslant\left(1-c\left\|S_{f}\right\|_{D}\right)^{-1}
$$

where $c=c(a)$ and $K\left(g_{j}\right)$ denotes the maximal dilatation of $g_{j}$.

Proof. Let

$$
h\left(r e^{i \theta}\right)=r^{a} e^{t(\theta-\log r)}
$$

for $r \in(0, \infty)$, and set $h(0)=0$ and $h(\infty)=\infty$. Then it is easy to verify that $h$ is a $K$ quasiconformal mapping of $\overline{\mathrm{C}}$, where $K=a+(2 / a)$, and that $h$ maps the imaginary axis onto $\beta$. Thus $\partial D_{j}=\beta$ is a $K$-quasiconformal circle. By the above mentioned theorem of Ahlfors, there exists a $\delta_{1}=\delta_{1}(a)$ such that each $f_{j}$ conformal in $D_{j}$ with $\left\|S_{f_{j}}\right\|_{D_{j}} \leqslant \delta_{1}$ has a quasiconformal extension $g_{3}$ to $\overline{\mathbf{C}}$, where

$$
\left\|\mu_{g_{j}}\right\|_{\infty} \leqslant c\left\|S_{f_{j}}\right\|_{D_{j}}\left(2-c\left\|S_{f_{j}}\right\|_{D_{j}}\right)^{-1}
$$

and $c=c(a)$. (For this last estimate see p. 22 in [10] or p. 132 in [2].)

Now suppose that $f$ satisfies the hypotheses of Lemma 6 and let $f_{j}=f \mid D_{j}$. Then since $\varrho_{D} \leqslant \varrho_{D_{j}}$ in $D_{j}$,

$$
\left\|S_{f_{j}}\right\|_{D_{j}} \leqslant\left\|S_{f}\right\|_{D} \leqslant \delta_{1}
$$

Thus $f$, has a quasiconformal extension $g$, to $\overline{\mathbf{C}}$ satisfying (21), and (19) follows directly.

Remark. If $f$ is conformal in $D$ with $\left\|S_{f}\right\|_{D} \leqslant \delta_{1}$, then Lemma 6 implies that $f_{j}=f \mid D_{j}$ has a homeomorphic extension to $D_{j} \cup\{0\}$ and hence that $f(z)$ has limits as $z \rightarrow 0$ in $D_{1}$ and as $z \rightarrow 0$ in $D_{2}$. We shall denote these limits by $f_{1}(0)$ and $f_{2}(0)$, respectively.

We require next the following consequence of a distortion theorem due to Teichmialler $[13]$. 
LEMMA 7. For each $\eta>0$ there exists a $K_{1}=K_{1}(\eta) \in(1, \infty)$ with the following property. If $g$ is a sense preserving quasiconformal mapping of $\overline{\mathbf{C}}$ with $K(g) \leqslant K_{1}$ and if $g$ fixes three points $z_{1}, z_{2}, \infty$, then

$$
|g(z)-z| \leqslant \eta\left|z_{1}-z_{2}\right|
$$

for $z$ with $\left|z-z_{1}\right|<\left|z_{1}-z_{2}\right|$.

Proof. Let $\varrho$ and $\sigma$ denote respectively the hyperbolic metric and distance in $G=\overline{\mathbf{c}}-$ $\{0,1, \infty\}$ and set

$$
b=\inf \{\varrho(z): z \in G \cap B\}, \quad B=\{z:|z| \leqslant 2\} .
$$

Then $\varrho$ is positive and infinitely differentiable in $G$ and $\varrho(z) \rightarrow \infty$ as $z \rightarrow 0$ or 1 . (See, for example, p. 51 and p. 246 in [12].) Hence $b \in(0, \infty)$. Set

$$
K_{1}=\exp (2 b \min (\eta, 1)) \in(1, \infty)
$$

Now suppose that $g$ is a sense preserving quasiconformal mapping of $\overline{\mathbf{C}}$ with $K(g) \leqslant K_{1}$, and suppose that $g$ fixes the points $0,1, \infty$. Then by the above mentioned theorem of Teichmüller,

$$
\sigma(g(z), z) \leqslant \frac{1}{2} \log K(g) \leqslant b \min (\eta, 1) \leqslant b
$$

for $z \in G$. (See pp, 29-31 in [13].) If $|z|<1$, then (23) implies that

$$
\sigma(g(z), z)=\inf \int_{\omega} \varrho d s \geqslant \inf \int_{\omega \cap B} b d s \geqslant b \min (|g(z)-z|, 2-|z|)
$$

where the infima are taken over all rectifiable arcs $\omega$ joining $z$ to $g(z)$ in $G$. Hence

$$
|g(z)-z|=\min (|g(z)-z|, 2-|z|) \leqslant \min (\eta, 1) \leqslant \eta
$$

for $|z|<1$ and we obtain (22) for the special case where $z_{1}=0$ and $z_{2}=1$. The general case then follows by applying what was proved above to the mapping

$$
h(z)=\frac{g\left(z\left(z_{2}-z_{1}\right)+z_{1}\right)-z_{1}}{z_{2}-z_{1}} .
$$

Remark. Lemma 7 also follows from a more elementary contra-positive normal family type argument. However this second method does not yield an explicit estimate for $K_{1}$ in terms of $\eta$.

LEMMA 8. For each $a \in(0,1 / 8 \pi)$ there exists a $\delta_{2}=\delta_{2}(a) \in\left(0, \delta_{1}\right]$ with the following property. If $f$ is conformal in $D$ with $\left\|S_{f}\right\|_{D} \leqslant \delta_{2}$ and if $f$ fixes $\infty$, then for $j=1,2, \alpha_{j}^{*}=f\left(\alpha_{j}\right)$ is a $b^{*}$-spiral onto $f_{j}(0)$ where $b^{*} \in(1,2)$. 
Proof. Set $c_{1}=c_{2}=\left(a^{2}+1\right)^{-\frac{1}{2}}, \quad d_{1}=d_{2}=a\left(a^{2}+1\right)^{-1}$,

$$
b=\frac{c_{1} c_{2}}{c_{1}^{2}-4 \pi d_{2}} \doteq(1-4 \pi a)^{-1} \in(1,2),
$$

and fix $b^{*} \in(b, 2)$. Next let $\varepsilon$ be as in Lemma 5 , set

$$
\eta=\frac{1}{6} \varepsilon r^{3}, \quad r=\frac{1}{2} \operatorname{dist}\left(1, \partial D_{1}\right)<\frac{1}{2}
$$

and choose $\delta_{2} \in\left(0, \delta_{1}\right]$ so that $\left(1-c \delta_{2}\right)^{-1} \leqslant K_{1}$, where $c=c(a)$ and $K_{1}=K_{1}(\eta)$ are as in Lemmas 6 and 7. Then $\delta_{2}$ depends only on $a$.

Now suppose that $f$ satisfies the hypotheses of Lemma 8. Then $\alpha_{1}^{*}=f\left(\alpha_{1}\right)$ and $\alpha_{2}^{*}=f\left(\alpha_{2}\right)$ are analytic open arcs with endpoints $f(1), f_{1}(0)$ and $f(-1), f_{2}(0)$ respectively. We shall show first that $\alpha_{1}^{*}$ is a $b^{*}$-spiral from $f(1)$ onto $f_{1}(0)$. By Proposition 2 we may assume without loss of generality that $f(1)=1$ and $f_{1}(0)=0$.

Let $g_{1}$ denote the quasiconformal extension of $f_{1}=f \mid D_{1}$ to $\overline{\mathbf{C}}$ given by Lemma 6 , fix $z_{1} \in \alpha_{1}$ and set

$$
h(z)=\frac{g_{1}\left(z_{1} z\right)}{g_{1}\left(z_{1}\right)}
$$

Then $h$ is a sense preserving quasiconformal mapping of $\overline{\mathbf{c}}, K(h) \leqslant K_{1}$ and $h$ fixes the points $0,1, \infty$. Hence

$$
|h(z)-z| \leqslant \eta
$$

for $|z-1|<1$ by Lemma 7 . Since $\varphi(z)=z_{1}, z$ maps $D_{1}$ onto $D_{1}, f\left(z_{1} z\right)=g_{1}\left(z_{1} z\right)$ for $z \in D_{1}$. Hence $h$ is analytic in $D_{1}$ and

$$
\left|\frac{z_{1} f^{\prime}\left(z_{1}\right)}{f\left(z_{1}\right)}-1\right|=\left|h^{\prime}(1)-1\right| \leqslant \frac{1}{2 \pi} \int_{\omega} \frac{|h(z)-z|}{|z-1|^{2}}|d z| \leqslant \frac{\eta}{r}<\varepsilon
$$

by (24), where $\omega$ is the positively oriented circle $\{z:|z-1|=r\}$. Similarly we obtain

$$
\left|\frac{z_{1}^{2} f^{\prime \prime}\left(z_{1}\right)}{f\left(z_{1}\right)}\right| \leqslant \frac{2 \eta}{r^{2}}<\varepsilon, \quad\left|\frac{z_{1}^{3} f^{\prime \prime \prime}\left(z_{1}\right)}{f\left(z_{1}\right)}\right| \leqslant \frac{6 \eta}{r^{3}}=\varepsilon .
$$

Then (5) and Lemma 5 imply that $\alpha_{1}^{*}$ is a $b^{*}$-spiral from 1 onto 0.

Next let $g(z)=f(-z)$. Then $g$ is conformal in $D$ with $\left\|S_{g}\right\|_{D} \leqslant \delta_{2}$ and $g(\infty)=\infty$. Hence $\alpha_{2}^{*}=g\left(\alpha_{1}\right)$ is a $b^{*}$-spiral by what was shown above and the proof is complete.

Lемма 9. For each $\varepsilon>0$ there exists a $\delta_{3}=\delta_{3}(a, \varepsilon) \in\left(0, \delta_{1}\right]$ with the following property. If $f$ is conformal in $D$ with $\left\|S_{f}\right\|_{D} \leqslant \delta_{3}$ and if $f$ fixes $1,-1, \infty$, then $\left|f_{1}(0)\right| \leqslant \varepsilon$ and $\left|f_{2}(0)\right| \leqslant \varepsilon$. 
Proof. Set $\eta=\min \left(\varepsilon / 4, \frac{1}{2}\right)$ and choose $\delta_{3} \in\left(0, \delta_{1}\right]$ so that $\left(1-c \delta_{3}\right)^{-2} \leqslant K_{1}$, where $c$ and $K_{1}$ are as in Lemmas 6 and 7. Then $\delta_{3}$ depends only on $a$ and $\varepsilon$.

Now suppose that $f$ satisfies the hypotheses of Lemma 9 and for $j=1,2$ let $g_{j}$ denote the quasiconformal extension of $f_{j}=f \mid D_{i}$ to $\overline{\mathbf{C}}$ given by Lemma 6 . Then $g=g_{2} \circ g_{1}^{-1}$ is a sense preserving quasiconformal mapping of $\overline{\mathbf{C}}$ with $K(g) \leqslant K_{1}$. If $z_{0} \in \beta-\gamma$, then for $j=1,2$, $z_{0} \in D_{j}$ and

$$
g_{j}\left(z_{0}\right)=\lim g_{j}(z)=\lim f_{j}(z)=f\left(z_{0}\right)
$$

where the limits are taken as $z \rightarrow z_{0}$ in $D_{j}$. Thus $g$ fixes points in $\beta-\gamma$ and hence, by continuity, the points $i,-i, \infty$. Thus

$$
\left|g_{2}(1)-1\right|=|g(1)-1| \leqslant 2 \eta, \quad 0<\left|g_{2}(1)+1\right| \leqslant 3
$$

by Lemma 7 . Set

$$
h(z)=\frac{2}{g_{2}(1)+1} g_{2}(z)-\frac{g_{2}(1)-1}{g_{2}(1)+1}
$$

Again $h$ is a sense preserving quasiconformal mapping of $\overline{\mathbf{c}}, K(h) \leqslant K_{1}$ and $h$ fixes $1,-1, \infty$. Thus $|h(0)| \leqslant 2 \eta$ by Lemma 7 and

$$
\left|f_{2}(0)\right|=\left|g_{2}(0)\right| \leqslant \frac{1}{2}\left|g_{2}(1)+1\right||h(0)|+\frac{1}{2}\left|g_{2}(1)-1\right| \leqslant \varepsilon
$$

Finally applying what was proved above to the mapping $-f(-z)$ yields the inequality $\left|f_{1}(0)\right| \leqslant \varepsilon$.

Proof of Theorem 2. Suppose that $a \in(0,1 / 8 \pi)$ and set

$$
\delta=\min \left(\delta_{2}(a), \delta_{3}\left(a, \frac{1}{5}\right)\right) \leqslant \delta_{1},
$$

where $\delta_{2}$ and $\delta_{3}$ are as in Lemmas 8 and 9. Next suppose that $f$ is conformal in $D$ with $\left\|S_{f}\right\|_{D} \leqslant \delta$. We shall show that $f(D)$ is not a Jordan domain. By following $f$ by a Möbius transformation, we may assume without loss of generality that $f$ fixes the points $1,-1, \infty$.

Now Lemma 8 implies that $\alpha_{1}^{*}=f\left(\alpha_{1}\right)$ and $\alpha_{2}^{*}=f\left(\alpha_{2}\right)$ are disjoint $b^{*}$-spirals from 1 onto $f_{1}(0)$ and from -1 onto $f_{2}(0)$, respectively, where $b^{*} \in(1,2)$. Next Lemma 9 implies that $\left|f_{1}(0)\right| \leqslant \frac{1}{5}$ and $\left|f_{2}(0)\right| \leqslant \frac{1}{5}$. Thus

$$
\left|f_{1}(0)-f_{2}(0)\right| \leqslant \frac{2}{5}<\frac{1}{b^{*}} \min \left(\left|1-f_{1}(0)\right|,\left|-1-f_{2}(0)\right|\right),
$$

and we conclude from Lemma 1 that $f_{1}(0)=f_{2}(0)$.

Next let $B=\{z:|z|<1\}$ and for $z \in B$ set $g(z)=h\left(\frac{i}{2}\left(z+\frac{1}{z}\right)\right)$, where $h$ is the quasicon. formal mapping of $\overline{\mathbf{C}}$ defined in (20) in the proof of Lemma 6 . Then $f \circ g$ is a quasicon- 
formal mapping of $B$ onto $f(D)$ and $f \circ g(z) \rightarrow f_{1}(0), f_{2}(0)$ as $z \rightarrow i,-i$ respectively in $B$. Hence $f(D)$ cannot be a Jordan domain, since otherwise $f \circ g$ would have a homeomorphic extension to $\bar{B}$ and $f_{1}(0) \neq f_{2}(0)$.

\section{References}

[1]. Ahufors, L. V., Quasiconformal reflections. Acta Math., 109 (1963), 291-301.

[2]. - Lectures on quasiconformal mappings. Van Nostrand Math. Studies 10, Princeton 1966.

[3]. —- Conformal invariants: topics in geometric function theory. McGraw-Hill, New York 1973.

[4]. Bers, L., On boundaries of Teichmüller spaces and on kleinian groups I. Ann. of Math., $91(1970), 570-600$.

[5]. - Universal Teichmüller space. Analytic methods in mathematical physics. Gordon and Breach (1970), 65-83.

[6]. - Uniformization, moduli, and kleinian groups. Bull. London Math. Soc., 4 (1972), 257-300.

[7]. — Quasiconformal mappings, with applications to differential equations, function theory and topology. Bull. Amer. Math. Soc., 83 (1977), 1083-1100.

[8]. Genrinc, F. W., Univalent functions and the Schwarzian derivative. Comm. Math. Helv., 52 (1977), 561-572.

[9]. GuggenheIMer, H. W., Differential geometry. McGraw-Hill, New York 1963.

[10]. LeHTo, O., Quasiconformal mappings in the plane. Lecture Notes 14, Univ. of Maryland 1975.

[11]. Nehari, Z., The Schwarzian derivative and schlicht functions. Bull. Amer. Math. Soc., $55(1949), 545-551$.

[12]. Nevanlinna, R., Eindeutige analytische Funktionen. Springer-Verlag, Berlin-GöttingenHeidelberg 1953.

[13]. TeichmüLler, O., Extremale quasikonforme Abbildungen und quadratische Differentiale. Abh. Preuss. Alsad. Wiss., 22 (1940), 1-197.

Received December 20, 1977 